\title{
The clinical value of two combination regimens in the Management of Patients Suffering from Covid-19 pneumonia: a single centered, retrospective, observational study
}

\author{
Ensieh Vahedi ${ }^{1}$ - Mostafa Ghanei ${ }^{1}$ - Ali Ghazvini ${ }^{1} \cdot$ Hossein Azadi ${ }^{1}$ - Morteza Izadi ${ }^{2}$ Yunes Panahi $^{3}$ - Saeid Fathi ${ }^{4}$. \\ Mahmood Salesi ${ }^{1}$ - Seyed Hassan Saadat ${ }^{5}$ - Amir Hossein Ghazale ${ }^{6}$ - Mohammad Rezapour ${ }^{6} \cdot$ Abolfazl Mozafari $^{7}$. \\ Nahid Zand ${ }^{8} \cdot$ Mohammadreza Raesi Parsaei $^{6} \cdot$ Mohammad Hossein Ranjkesh $^{6} \cdot$ Ramezan Jafari $^{9}$. \\ Fatemeh Movaseghi ${ }^{7} \cdot$ Enayat Darabi $^{10}$
}

Received: 27 March 2020 / Accepted: 2 June 2020 / Published online: 19 June 2020

(C) Springer Nature Switzerland AG 2020

\begin{abstract}
Background There is no identified pharmacological therapy for COVID-19 patients, where potential therapeutic strategies are underway to determine effective therapy under such unprecedented pandemic. Therefore, combination therapies may have the potential of alleviating the patient's outcome. This study aimed at comparing the efficacy of two different combination regimens in improving outcomes of patients infected by novel coronavirus (COVID-19).

Methods This is a single centered, retrospective, observational study of 60 laboratory-confirmed COVID-19 positive inpatients ( $\geq 18$ years old) at two wards of the Baqiyatallah Hospital, Tehran, Iran. Patient's data including clinical and laboratory parameters were recorded. According to the drug regimen, the patients were divided into two groups; group I who received regimen I consisting azithromycin, prednisolone, naproxen, and lopinavir/ritonavir and group II who received regimen II including meropenem, levofloxacin, vancomycin, hydroxychloroquine, and oseltamivir.

Results The oxygen saturation $(\mathrm{SpO} 2)$ and temperature were positively changed in patients receiving regimen I compared to regimen II $(P=0.013$ and $P=0.012$, respectively). The serum level of C-reactive protein (CRP) changed positively in group I $(P<0.001)$. Although there was a significant difference in platelets between both groups $(75.44$ vs $51.62, \mathrm{P}<0.001)$, their change did not clinically differ between two groups. The findings indicated a significant difference of the average length of stay in hospitals (ALOS) between two groups, where the patients under regimen I showed a shorter ALOS $(6.97 \mathrm{vs} 9.93, P=0.001)$. Conclusion This study revealed the beneficial effect of the short-term use of low-dose prednisolone in combination with azithromycin, naproxen and lopinavir/ritonavir (regimen I), in decreasing ALOS compared to regimen II. Since there is still lack of evidence for safety of this regimen, further investigation in our ongoing follow-up to deal with COVID-19 pneumonia is underway.
\end{abstract}

Keywords Coronavirus disease $2019 \cdot$ Combination therapy · Length of stay in hospitals (ALOS)

Mostafa Ghanei

mghaneister@gmail.com

1 Chemical Injuries Research Center, Systems Biology and Poisoning Institute, Baqiyatallah University of Medical Sciences, Tehran, Iran

2 Health Research Center, Baqiyatallah University of Medical Sciences, Tehran, Iran

3 Faculty of pharmacy, pharmacotherapy department, Baqiyatallah University of Medical Sciences, Tehran, Iran

4 University of Tehran, Tehran, Iran

5 Behavioral sciences research center, Lifestyle institute, Baqiatallah University of Medical Sciences, Tehran, Iran
6 Student Research committee, Baqiyatallah University of medical Sciences, Tehran, Iran

7 Department of Medical Science, Qom Branch, Islamic Azad University, Qom, Iran

8 Department of Internal Medicine, Qom university of medical sciences, Qom, Iran

9. Department of Radiology and Chemical Injury Research Center, Baqiyatallah University of Medical Sciences, Tehran, Iran

10 School of Public Health, Tehran University of Medical Sciences, Tehran, Iran 


\section{Introduction}

Coronavirus disease 2019 (COVID-19) is a viral disease caused by the novel coronavirus 2019 (nCOV-19), which is known as a positive-sense single-strand RNA segment with an approximate weight of $27-32 \mathrm{~kb}$, belonging to a unique clade of the betacoronaviruses $[1,2]$.

The World Health Organization (WHO) stated a public health emergency of international concern after the rapid spread of the COVID-19 on January 30, 2020, and it was presented as a pandemic on March 11, 2020 as many regions of the world had been affected by then. COVID-19 infection also spread across Iran with an increasing pace [3], where has affected a large population. So far, there has been no international complete consensus or guideline on any specific antiCOVID-19 treatments.

For the diagnosis of COVID-19, chest computed tomography (CT) has been suggested as a sensitive imaging technique for early diagnosis of COVID-19 pneumonia in combination with patients' clinical symptoms and laboratory findings $[4,5]$.

The association of pathophysiology of human coronavirus infection with inflammatory reactions and the consequent cytokine storm has previously been described $[6,7]$. Furthermore, dyspnea and hypoxemia suggest occurrence of a severe pneumonia [8], leading to acute respiratory distress syndrome (ARDS) [9]. Accumulative evidence has revealed organizing pneumonia patterns based on chest $\mathrm{CT}$ scans and pathological findings in some patients with COVID-19 $[5,6,10]$. It is suggested that COVID-19 induces diverse pathophysiological processes in two-phase immune response including immune defense-based protective phase (non-severe stage) and the second inflammation-driven damaging phase [11]; thus tailored therapeutic strategies need to be recommended.

A growing body of evidence has indicated presence of a cytokine storm syndrome in patients suffering from severe COVID-19 [12], in which good general health may not be beneficial [11].

In cytokine storm syndrome a series of immune responses are generated resulting in alteration of peripheral white blood cells (WBCs) and immune cells (e.g., T and B lymphocyte, macrophage, etc.) [13], which lead to the secretion of proinflammatory cytokines and the consequent severe lung injury. Therefore, expeditious anti-inflammatory treatment in an early phase might be effective in controlling the disease among selective patients [14].

There is active controversy on the use of corticosteroids in the second phase that is not routinely considered as a therapeutic approach in such conditions (i.e., $\mathrm{SpO} 2<90 \%$ ) and COVID-19 lung injuries [15-17], but their timely prescription at a proper dose may inhibit disease progression or deterioration [6]. Immunomodulation therapy has been considered as a therapeutic strategy for treatment of hyperinflammation which may reduce the mortality rate [12]. A retrospective, multicenter study in Wuhan, China demonstrated that mortality rate of COVID-19 could be probably linked to virally-driven hyperinflammation [18], indicating efficacy of corticosteroids in reducing hyperinflammation and immunosuppression [12].

Hypothetically, use of a combination regimen of off-label medications (i.e., azithromycin, low-dose prednisolone, naproxen, and lopinavir/ritonavir (Kaletra) may be effective in the management and control of the disease. Macrolides are indicated for different respiratory infectious diseases, and so azithromycin may be beneficial in fighting COVID-19 with its therapeutic value (e.g., antiviral effect) [19].

In addition, corticosteroids as a double-edged sword (i.e., the effect of prednisolone on 5-lipoxygenase activity) are considered to have anti-inflammatory properties and nonsteroidal anti-inflammatory drugs (NSAIDs; e.g., naproxen) also used for their antipyretic, analgesic and anti-inflammatory effects. From the other point of view, low-dose, short-term administration of prednisolone may be capable of reducing potential risks of corticosteroid therapy [20]. Although there seems to exist no strong evidence of any specific treatment strategy for COVID-19 infection, immune-boosting therapies (e.g., antisera or pegylated IFN $\alpha$, etc.) may be of great importance in the initial phase as well as an appropriate general health and genetic background (e.g., HLA) that may be implicated in the specific endogenous antiviral immunity; also, immunosuppressive and antiviral strategies may be applicable in the inflammatory phase [11].

Currently, there is limited evidence for effective therapeutic strategies and the comprehensive data on clinical randomized trials are lacking and most of the medications are used as off-label or compassionate use. The physicians may indicate a medication or a combination of medications as clinical practice and based on their responsibility or may investigate on potential off-label medications [21]. Thus, RCTs are ongoing all over the world for many therapeutic agents and strategies such as remdesivir, chloroquine, hydroxychloroquine, favipiravir, and corticosteroids, as well as convalescent plasma transfusion, etc.

Based on hospital data repository, and according to the guideline issued by the Iranian Ministry of Health and Medical Education [22], in which a three-drug regimen including oseltamivir, hydroxychloroquine, and lopinavir/ ritonavir were recommended for inpatients, some patients in Baqiyatallah hospital received azithromycin, prednisolone, naproxen, and lopinavir/ritonavir and we assigned them to group I and the other patients received oseltamivir, and hydroxychloroquine and we assigned them to group II. It is noteworthy that two different drug regimens were used at two different wards of hospital as prescribed by two groups of pulmonologist and infectious disease specialists.

Therefore, the present study aimed to evaluate short-term effects of regimen I (azithromycin, prednisolone, naproxen, 
and lopinavir/ritonavir) in group I patients in comparison to regimen II (meropenem, levofloxacin, vancomycin, hydroxychloroquine, and oseltamivir) in group II patients in the treatment of COVID-19 infection.

\section{Material and methods}

\section{Study design}

We conducted a single centered, retrospective, observational studybetween February 29, 2020 and March 23, 2020 at two wards of Baqiyatallah Hospital, Tehran, Iran, where two different drug regimens were used by infectious disease specialists and pulmonologists for the treatment of patients suffering from COVID-19. The patients in the group I were given a four-drug combination regimen (azithromycin [250 mg/daily], prednisolone [25 mg/daily], naproxen [250 $\mathrm{mg}$ twice a day], and lopinavir/ritonavir [200/50 mg tablets, two times/ $12 \mathrm{~h}$ ]) as regimen I. It is worth noting that lopinavir/ritonavir was given as a combined regimen according to the guidelines issued by the Iranian Ministry of Health and Medical Education [22]. The patients in group II, received regimen II including meropenem ([1 g/8 h), levofloxacin (500 mg daily), vancomycin (1 g/12 h), hydroxychloroquine (200 mg/12 h), and oseltamivir (75 mg/12 h).

\section{Data collection}

The laboratory-confirmed COVID-19 inpatients ( $\geq 18$ years old) with moderate disease who admitted in two wards of Baqiyatallah hospital, retrospectively enrolled in the study. This hospital was one of the hospitals dedicated to COVID-19 patients in Tehran, Iran. Our study was designed according to the national and international ethical guidelines and was approved by the Ethics Committee of Baqiyatallah University of Medical Sciences with the code of IR.BMSU.REC.1398435. The informed consent was obtained from the patients for using their medical records. Additionally, patient confidentiality was considered by protecting the electronic data in computer.

The patients' medical information including demographic data, COVID-19 test using a real-time RT-PCR via throatswab specimens, clinical features, routine laboratory tests, chest CT scans (before and after discharge) according to the WHO interim guidance [23], treatment measures, comorbidities and data on the outcomes were extracted independently by two physicians through a standard case record form provided by the hospital. It should be noted that laboratory tests were requested based on the physicians order including $\mathrm{C}$-reactive protein (CRP) concentration and complete blood count as the time-series data before and after the treatment depending on the duration of hospitalization. Daily vital signs monitoring, including body temperature, heart rate, respiratory rate, blood pressure, and $\mathrm{SpO} 2$, were also recorded from patients medical information. The records indicate that other supportive measures including active control over high fever (paracetamol) and supplemental oxygen have been considered for all patients if necessary.

The patient's definitions of clinical outcomes including moderate disease (having fever, respiratory symptoms, radiological sign of mild pneumonia, no complications and severe conditions) and severe disease (having respiratory distress, resting $\mathrm{SpO} 2<93$, and rapid disease progression on $\mathrm{CT}$ scan) were also extracted.

Improved outcomes were defined as subsided fever, improved COVID-19 pneumonia (confirmed via CT scans), as well as improvements in symptoms of the upper respiratory system, while failed outcomes of the patients were determined as progression toward a critical condition or death. Finally, data adjudication was performed by a pulmonologist. According to the hospital data, two diabetic inpatients received treatment at two wards of hospital after adjusting their drug doses for controlling their diabetes (in both groups I and II).

\section{Statistical analysis}

Descriptive statistics were reported as frequencies and percentages or as the mean \pm standard deviation or confidence Interval 95\% (CI95\%). The T- test and MannWhitney $U$ were used for normally-distributed variables (evaluated by the one-sample Kolmogrov Smirnov test) and non-normally distributed variables, respectively. In addition, the Chi-square test was used to compare the difference between the percentages of variables between the two groups. The Generalized Estimating Equations (GEE) regression was applied for analyzing repeated measures. The probability value of 0.05 or less $(P \leq 0.05)$ was set to determine the significance level.

\section{Results}

\section{Demographics and clinical characteristics}

By March 23, 2020, 60 laboratory-confirmed COVID-19 inpatients were enrolled in the study. The demographic data and base line variables are presented in Table 1. The mean age of the patients was 59.33 years $(S D=14.40)$ in group I and 57.46 years $(\mathrm{SD}=12.74)$ in group II. Furthermore, the study included $25(41.66 \%)$ males and $35(58.33 \%)$ females, and two patients with underlying diseases, (diabetes), in group I and II $(3.33 \%)$ were treated after adjusting their drug doses for control of diabetes. 
Table 1 The distribution of demographic variables and base line variables between two groups

\begin{tabular}{|c|c|c|c|c|c|}
\hline \multirow[t]{3}{*}{ Categorical Variables } & \multicolumn{4}{|l|}{ Group } & \multirow{3}{*}{$P$ value } \\
\hline & \multicolumn{2}{|c|}{ Regimen I } & \multicolumn{2}{|c|}{ Regimen II } & \\
\hline & $\mathrm{N}$ & $\%$ & $\mathrm{~N}$ & $\%$ & \\
\hline Gender (Male) & 11 & $36.7 \%$ & 14 & $46.7 \%$ & 0.600 \\
\hline Smoke (Yes) & 2 & $6.7 \%$ & 1 & $3.3 \%$ & 0.612 \\
\hline Continuous Variables & Mean & Standard Deviation & Mean & Standard Deviation & $\mathrm{P}$ value \\
\hline Age & 59.33 & 14.40 & 57.46 & 12.74 & 0.612 \\
\hline BMI & 28.65 & 4.20 & 28.73 & 6.19 & 0.954 \\
\hline Temperature & 37.62 & 0.82 & 37.52 & 0.68 & 0.585 \\
\hline Lymphocyte & 21.52 & 9.38 & 22.99 & 8.9 & 0.554 \\
\hline $\mathrm{SPo} 2$ & 86.7 & 8.75 & 83.17 & 10.16 & 0.233 \\
\hline PLT & 201.92 & 83.24 & 173.57 & 48.44 & 0.121 \\
\hline CRP & 79.47 & 53.66 & 50.43 & 31.7 & 0.019 \\
\hline WBC & 7.59 & 3.08 & 5.58 & 1.54 & 0.006 \\
\hline PR & 90.15 & 11.4 & 91.88 & 11.97 & 0.568 \\
\hline RR & 18.98 & 2.94 & 18.4 & 1.85 & 0.362 \\
\hline SBP & 122.52 & 10.92 & 123.65 & 12.61 & 0.714 \\
\hline DBP & 77.48 & 5.64 & 76.65 & 8.96 & 0.672 \\
\hline
\end{tabular}

SBP: Systolic Blood pressure, DBP: Diastolic Blood pressure, RR: Respiratory Rate, PR: Pulse Rate, WBC: White Blood Cell, CRP: C - reactive protein, PLT: Platelet Count

$\mathrm{P}$ value was calculated by chi square, t-test or Mann-Whitney test

The most common symptoms on admission were recorded as fever, dry cough, myalgia or fatigue and shortness of breath. Two-thirds of the patients experienced anorexia and headaches as mentioned in their medical records.

The main clinical parameters are presented herein. The patients' body temperature was recorded, followed by their resting oxygen saturation $(\mathrm{SpO} 2)$, respiratory rate $(\mathrm{RR})$, pulse rate (PR), systolic blood pressure (SBP), and diastolic blood pressure (DBP).

The body temperature showed a statistically significant change in group I in day 3 compared to baseline $(P<0.001$; Table 2), while the change was not significant in group II (37.62-37.02 vs $37.52-37.28)$.

Our results showed that the mean change of RR and PR exhibited no significant change in both groups compared to baseline (Table 2). Additionally, the changes of SBP and DBP were not found to be statistically significant in both groups (Table 2).

Furthermore, the mean change of $\mathrm{SpO} 2$ was significantly noticeable in patients receiving regimen I compared to regimen II $(p=0.011$ vs $P=0.527)$ (Table 2$)$.

\section{Laboratory findings}

On admission, white blood cells (WBCs) were reported to be lower than the normal range in $12(20 \%)$ patients (WBCs less than $4 \times 109 / \mathrm{L})$ and above the normal range in $48(80 \%)$ patients. Lymphocytes (LYMs) were found to be lower than the normal range in $26 \%$ of patients (16 patients; lymphopenia) on admission time and above the borderline or normal range in $74 \%$ of patients (44 patients). Additionally, the hematologic assessment of patients revealed highly decreased platelets (PLTs) for 17 patients (28.33\%) on admission. The mean change of laboratory parameters including WBCs, LYMs, PLTs and CRP are presented in Table 2.

Of 60 patients enrolled in this study, the mean concentration of CRP was significantly decreased in group I compared to group II $(p<0.001)$ (Table 2). Additionally, platelet counts increased relatively in both groups, but the changes were relatively distinctive for group I patients in comparison to group II, where a statistically significant difference was observed between the two groups $(\mathrm{p}<0.001)$ (Table 2).

There was also no statistically significant difference in the WBCs between groups $(p=0.131)$ and the mean circulating LYMs did not vary significantly between the groups after the treatment $(p=0.961)$ (Table 2).

\section{The average length of stay in hospitals (ALOS)}

The patients in group I responded more effectively to the fourdrug combination regimen I compared to group II, as manifested by ALOS in group I, 6.97 days $(\mathrm{SD}=3.08)$ compared to group II with mean ALOS of 9.93 days $(\mathrm{SD}=3.16)(P=$ $0.001)$. In other words, ALOS was significantly lower in the 
Table 2 The GEE regression results for the variables during study period

\begin{tabular}{|c|c|c|c|c|c|c|c|c|}
\hline \multirow[t]{2}{*}{ Variables } & \multirow[b]{2}{*}{ Group } & \multicolumn{2}{|l|}{ Mean } & \multirow{2}{*}{$\begin{array}{l}\text { Mean Difference } \\
\text { (day 3-base line) }\end{array}$} & \multirow[t]{2}{*}{ Standard Error } & \multicolumn{2}{|l|}{$95 \% \mathrm{CI}$} & \multirow[t]{2}{*}{$\mathrm{p}$ value } \\
\hline & & base line & day 3 & & & Lower & Upper & \\
\hline \multirow[t]{3}{*}{ Temperature } & Regimen I & 37.62 & 37.02 & -0.60 & 0.16 & -0.90 & -0.29 & $<0.001$ \\
\hline & Regimen II & 37.52 & 37.28 & -0.24 & 0.13 & -0.49 & 0.01 & 0.061 \\
\hline & & & $\mathrm{p}$ value & 0.012 & & & & \\
\hline \multirow[t]{3}{*}{ Lymphocyte } & Regimen I & 21.52 & 22.11 & 0.59 & 1.75 & -2.83 & 4.01 & 0.737 \\
\hline & Regimen II & 22.99 & 23.49 & 0.50 & 1.78 & -2.98 & 3.98 & 0.777 \\
\hline & & & $\mathrm{p}$ value & 0.961 & & & & \\
\hline \multirow[t]{3}{*}{$\mathrm{SpO} 2$} & Regimen I & 86.70 & 89.75 & 3.05 & 1.20 & 0.69 & 5.40 & 0.011 \\
\hline & Regimen II & 83.17 & 83.68 & 0.51 & 0.81 & -1.07 & 2.09 & 0.527 \\
\hline & & & $\mathrm{p}$ value & 0.013 & & & & \\
\hline \multirow[t]{3}{*}{ PLT } & Regimen I & 201.92 & 277.36 & 75.44 & 12.74 & 50.47 & 100.41 & $<0.001$ \\
\hline & Regimen II & 173.57 & 225.19 & 51.62 & 15.805 & 20.64 & 82.60 & 0.001 \\
\hline & & & $\mathrm{p}$ value & $<0.001$ & & & & \\
\hline \multirow[t]{3}{*}{ CRP } & Regimen I & 79.47 & 24.04 & -55.43 & 10.82 & -76.63 & -34.23 & $<0.001$ \\
\hline & Regimen II & 50.43 & 45.79 & -4.64 & 8.59 & -21.47 & 12.19 & 0.589 \\
\hline & & & $\mathrm{p}$ value & $<0.001$ & & & & \\
\hline \multirow[t]{3}{*}{ WBC } & Regimen I & 7.59 & 6.47 & -1.12 & 0.74 & -2.57 & 0.32 & 0.128 \\
\hline & Regimen II & 5.58 & 5.38 & -0.20 & 0.45 & -1.07 & 0.67 & 0.652 \\
\hline & & & $\mathrm{p}$ value & 0.131 & & & & \\
\hline \multirow[t]{3}{*}{ PR } & Regimen I & 90.15 & 85.14 & -5.01 & 2.10 & -9.12 & -0.90 & 0.017 \\
\hline & Regimen II & 91.88 & 83.06 & -8.82 & 2.04 & -12.82 & -4.82 & $<0.001$ \\
\hline & & & $\mathrm{p}$ value & 0.066 & & & & \\
\hline \multirow[t]{3}{*}{ RR } & Regimen I & 18.98 & 17.94 & -1.04 & 0.60 & -2.20 & 0.13 & 0.082 \\
\hline & Regimen II & 18.40 & 17.96 & -0.44 & 0.34 & -1.11 & 0.23 & 0.201 \\
\hline & & & $\mathrm{p}$ value & 0.22 & & & & \\
\hline \multirow[t]{3}{*}{ SBP } & Regimen I & 122.52 & 118.87 & -3.65 & 2.08 & -7.73 & 0.43 & 0.08 \\
\hline & Regimen II & 123.65 & 117.58 & -6.07 & 2.76 & -11.48 & -0.67 & 0.028 \\
\hline & & & $\mathrm{p}$ value & 0.321 & & & & \\
\hline \multirow[t]{3}{*}{ DBP } & Regimen I & 77.48 & 76.62 & -0.86 & 1.07 & -2.97 & 1.24 & 0.422 \\
\hline & Regimen II & 76.65 & 72.86 & -3.79 & 2.04 & -7.79 & 0.22 & 0.064 \\
\hline & & & $\mathrm{p}$ value & 0.073 & & & & \\
\hline
\end{tabular}

SBP: Systolic Blood pressure, DBP: Diastolic Blood pressure, RR: Respiratory Rate, PR: Pulse Rate WBC: White Blood Cell, CRP: C - reactive protein, PLT: Platelet Count

patients receiving regimen I when compared with those receiving regimen II (Table 3). Additionally, the median time from admission to discharge was 6 and 10 days for regimens I and II, respectively (IQR $=4-9.25$ vs 7-12). No patient was admitted to the intensive care unit (ICU), but an 86 years old female patient in group II died of respiratory failure.

\section{CT findings}

The researchers carefully assessed the CT findings of the enrolled patients before and after the treatment. Depending on their hospitalization time, abnormal chest CT scan features were observed in all patients on admission.

Table 3 The comparison of hospitalization length between two groups

\begin{tabular}{|c|c|c|c|c|c|c|c|}
\hline \multirow[t]{2}{*}{ Group } & \multirow[t]{2}{*}{ Mean } & \multirow[t]{2}{*}{ Standard Deviation } & \multirow[t]{2}{*}{ Median } & \multirow[t]{2}{*}{$\mathrm{IQR}^{*}$} & \multicolumn{2}{|c|}{$95 \% \mathrm{CI}$} & \multirow[t]{2}{*}{$\mathrm{P}$ value } \\
\hline & & & & & Lower & Upper & \\
\hline Regimen I & 6.97 & 3.08 & 6.5 & $4-9.25$ & 5.82 & 8.12 & 0.001 \\
\hline Regimen II & 9.93 & 3.16 & 10 & $7-12$ & 8.75 & 11.11 & \\
\hline
\end{tabular}

*interquartile range (IQR) 
On admission, chest CT images of inpatients showed bilateral lung involvement representing diffuse lesions, bilateral ground-glass opacity appearance, and subsegmental areas of consolidative opacities (Fig. 1A; Fig. 2 A and C). However, complete resolution of opacities was recorded for these patients 10 days after the treatment with regimen I, suggesting a dramatic response to the treatment without complications (Fig. 1B; Fig. 2B and D). In group II, small patchy ground glass opacities on both lungs fields were observed on the admission day (Fig. 3A and B), followed by multifocal bilateral consolidation and severe lung involvement (Fig. $3 \mathrm{C}$ and D).

\section{Discussion}

This report is an observational study of hospitalized patients with COVID-19 and aimed to compare short-term effects of two combination regimens I and II according to hospital data.

Based on the hospital data, azithromycin, prednisolone, naproxen, and lopinavir/ritonavir (regimen I) have been considered by physicians for blocking the inflammatory cascade and combating virus in early infection detection based on the two-phase immune responses, because a combination therapy may be capable of halting the pathologic process. From the point of view of anti-inflammatory treatment, immunosuppressive strategies [24] or symptomatic management should be considered for suppressing inflammation in inflammation-driven damaging phase coupled with antiviral therapies. Furthermore, higher viral loads in asymptomatic and paucisymptomatic (minimally symptomatic) patients (over the first days) has been detected in the upper respiratory tract of COVID-19 patients [25, 26], except for the patients with critical disease as having different viral kinetics (a persistent and high viral excretion), [25], suggesting their role in disseminating the disease and difference in viral shedding pattern or viral loads $[25,26]$ in comparison with SARS-CoV [27]. This evidence may affect not only control measures, but also therapeutic strategies.

It has been reported that low-dose short-term administration of prednisolone may be capable of reducing potential risks of corticosteroid therapy [20]. There are some evidence showing some degrees of effectiveness of short-term low-tomoderate-dose corticosteroids therapy in combination with immunoglobulin in decreasing lung injury, normalizing body temperature, CRP levels, lymphocyte counts, and SpO2 levels, leading to inhibition of inflammation [28], which is more or less in agreement with our findings revealed by immunomodulatory theraphy (regimen I). Short-term and lowdose administration of corticosteroids has been prudently recommended for critically ill patients suffering from COVID-19 (e.g., ARDS, refractory septic shock, and chronic obstructive pulmonary disease, etc.) [29]. While corticosteroids were not routinely recommended for treatment of COVID-19 patients, a retrospective cohort study of COVID-19 in Wuhan, China reported the benefits of methylprednisolone by decreasing the risk of death due to COVID-19 in patients who developed ARDS [10]. In contrast, corticosteroid use was found to be linked to risk of death, secondary bacterial infections and longer ALOS, as reveled by meta-analysis [30, 31]. The efficacy and duration of corticosteroid use in COVID-19 patients requires further investigation by retrospective studies and RCTs as conflicting results have been reported due to heterogeneity of studies and timings of use, etc. Numerous RCTs such as

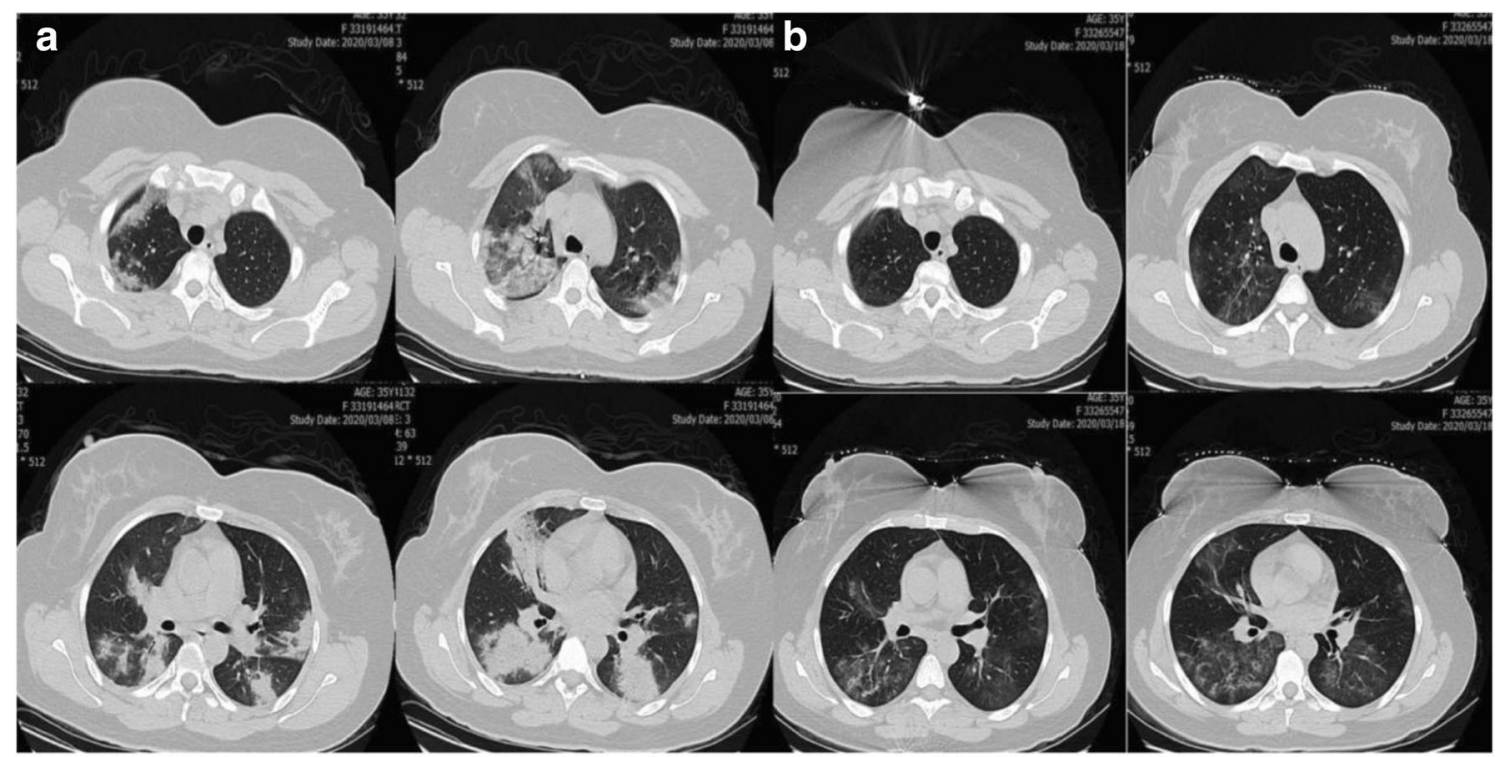

Fig. 1 Axial non-contrast CT scan in a 35-year-old female with COVID-19 pneumonia

A: Patchy consolidative opacities in both lungs are indicated; B: ten day later nearly complete resolution of opacities and dramatic response to regimen I treatment was revealed for patient. 
Fig. 2 Axial non contrast CT scan of two 75-year-old women with covid-19 pneumonia. A: Patchy ground glass opacities; B: significant resolution of ground glass opacities is notable ten-day after treatment with regimen I
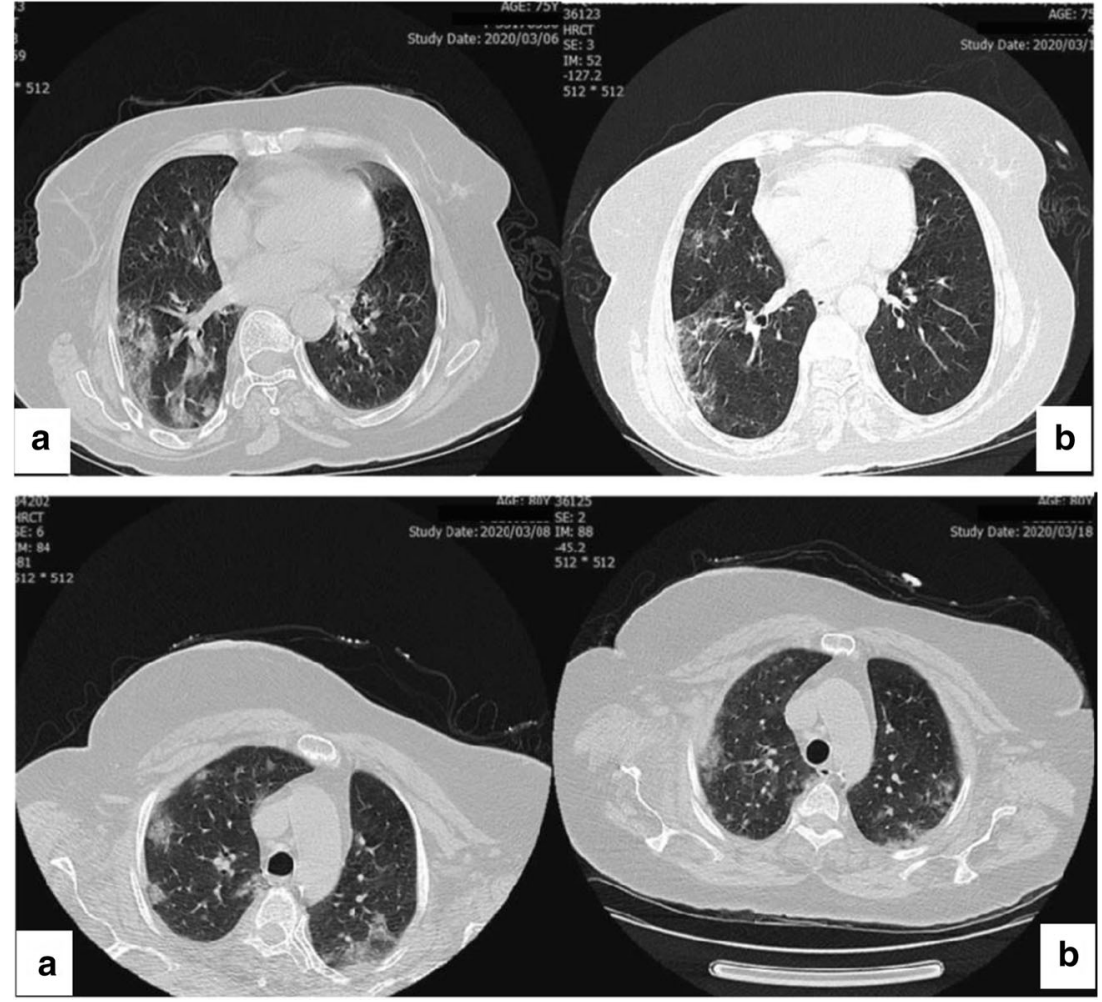

ChiCTR2000029386, NCT04244591, ChiCTR2000030481, and ChiCTR2000029656 in COVID-19 are underway to determine the clinical value of corticosteroid therapy for preventing hyperinflammation, and decreasing the risks of secondary bacterial infections, etc. Likewise, blocking IL-6, IL-1, TNF and cytokine licensed- mesenchymal stem cell therapies are among other strategies that may be beneficial for treatment of COVID-19 patients [11,32].
Lopinavir/ritonavir and arbidol have also been reported to be beneficial in treatment of COVID-19 patients [6]. While lopinavir/ritonavir use was not associated with clinical improvement beyond standard care in patients suffering from severe COVID-19 [33] due to particularly challenging population and/or lack of lopinavir potency against COVID-19, its beneficial effect for some secondary endpoints was revealed, where the safety of this therapeutic approach was achieved
Fig. 3 Axial chest CT scan images without contrast (a 40year-old women). Small patchy ground glass opacities on both lungs field on the admission day (A-B), and eleven day later multifocal bilateral consolidation and sever lung involvement (C-D) is noted in patient receiving regimen II
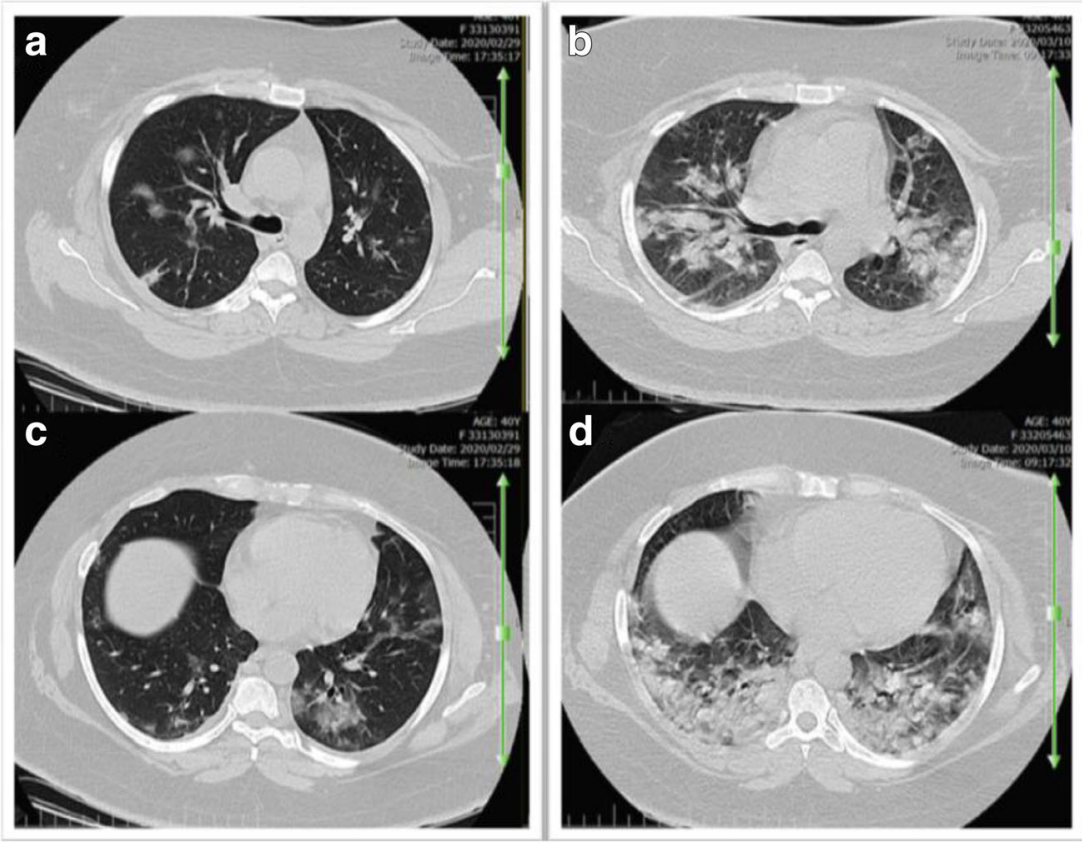
[34]. Furthermore, the secondary endpoints provided both lower number of death (hope) and lack of discernible effect on viral shedding (discouragement) [35]. Therefore, further studies are needed to evaluate lopinavir/ritonavir as monotherapy or combination therapy for clinical improvement.

There is no conclusive evidence that NSAIDs are certainly contraindicated for COVID-19, while naproxen, with its wellknown anti-inflammatory, ant-influenza [36] and antiplatelet properties in combination with antiviral agents may be potentially useful; however, no conclusive evidence demonstrated its risk for COVID-19 patients in clinical trial (CT04325633) or other respiratory infections [37].

Empirical use of broad-spectrum antibiotics requires urgent de-escalation, but difficulties in differentiating bacterial and viral pneumonias and time-consuming laboratory tests have led to their empirical use in the critical conditions. The use of therapeutic combination regimen including meropenem, levofloxacin, vancomycin, hydroxychloroquine, and oseltamivir could be hypothetically capable of fighting COVID-19, especially in the case of antiviral effect of oseltamivir and the immunomodulatory effect of hydroxychloroquine. Currently, there is no strong evidence for the use of oseltamivir and accumulating evidence does not considerably support the clinical benefit of hydroxychloroquine in COVID-19 patients, while mobile cardiac outpatient telemetry is recommended due to cardiovascular risks of this antimalarial drug [38]. Therefore, the optimal therapeutic use of this medication has yet to be clarified by clinical trials.

Clinical manifestation of the disease was found to be more effectively resolved in group I patients who received the combination regimen I compared to group II patients who received the combination regimen II. Our data revealed that the $\mathrm{SpO} 2$ had a relative increased pattern in group I patients receiving regimen I in comparison to those in group II, indicating that regimen I had a positive significant impact on improvement of $\mathrm{SpO} 2$, while the patients in group II did not show clinical significant improvement. It should be taken into account that the mean changes of $\mathrm{SpO} 2$ for the third, fourth, and fifth days of admission were noticeable for regimen I.

The differences in daily body temperature between the groups were found to be significant; but the relative drop in fever in group I, may be attributable partly to NSAID use (naproxen). The mean changes of RR, PR, and blood pressure (SBP and DBP) did not vary between two groups after the treatment, but relatively (not statistically significant) rapid improvement of respiratory rate was seen in group I patients compared to group II.

The laboratory findings showed an increased level of CRP concentration on admission in patients with COVID-19, as previously reported for betacoronavirus infections $[5,39$, 40]. Furthermore, persistently increased CRP level is a strong index for the continuation of inflammation, suggesting provision of additional therapeutic interventions [41]. A declining trend in CRP values was detected 3 days after starting the treatment, when a reduction in fever was also observed. Laboratory tests indicated that the mean concentration of CRP significantly decreased in group I compared to group II.

Decreased total LYMs and decreasing trend in LYMs until death was found to be the most common laboratory findings on admission time, indicating the probability of the association between COVID-19 and cellular immune deficiency and presence of persistent lymphopenia [42]. COVID-19 is more likely to affect $\mathrm{T}$ lymphocytes (CD4 and CD8 cell depletion), as does severe acute respiratory syndrome-related coronavirus (SARS-CoV) [10]. Inaddition, decrease in CD3, CD4, and CD8 $T$ cells have also been observed from the early phases of COVID-19 to the recovery [10] as demonstrated for SARS-CoV in the peripheral blood $[43,44]$. T cell responses are capable of suppressing the overactivation of innate immunity [45] and gradual elevation of lymphocyte responses may be of great importance for effective immunity responses against COVID-19 [15].

In this study, no statistically significant difference was found in mean changes of WBCs count between both groups.

As decreased platelet count is used for screening of hyperinflammation along with other laboratory tests (e.g., a rise in ferritin as an acute phase reactant, $\mathrm{H}$ Score for secondary HLH and ESR), may be important to identify subgroup of severe COVID-19 patients who may benefit from immunosuppression (i.e., improvements in mortality rate) [12].

On admission, depressed platelet counts were detected in COVID-19 patients, while both combination regimens relatively revived platelet counts; however, no significant clinical difference was found between two groups compared to the baseline data.

Regimen I proved efficient in improving the clinical outcomes of COVID-19 patients by addressing a shorter patient's hospital stay in our patients in group I. Furthermore, we observed the beneficial effects of regimen I on CT scan of the patients by comparing before and after results. It seems to us that the capacity of the four drugs regimen including azithromycin, naproxen, prednisolone and lopinavir/ritonavir could be effectively considered for the management of COVID-19 pneumonia, regarding overall cost savings due to reduced ALOS and decrease in antibiotic use.

In the next step, we recommend further studies to focus on the problems of widespread ineffective antiviral use by physicians for management of the patients. Therapeutic options such as steroids are in need of further elucidation for COVID19-induced lung injuries. Additionally, the evidence for potential harm or benefit (i.e., safety and efficacy) of azithromycin and naproxen in the case of COVID-19 seems to be of paramount clinical importance. Therefore, an ongoing follow-up of adverse post-treatment outcomes is underway to provide sufficient evidence for the overall harm or benefit of the current combination therapies. 
In conclusion, we assume that the patients exhibited better outcomes in the four-drug combination (regimen I) than the regimen II in parallel, in terms of decrease in CRP, increase in platelet counts, and improvement of $\mathrm{SpO}$. This effect was due to immunomodulatory properties, antibiotic with triple effects (azithromycin) and antiviral effects in patients without comorbidity conditions, except for one diabetic patients, in the second inflammation-driven damaging phase as rescue protocol; however, those changes were not clinically significant despite their statistically significant difference, compared to base line. A significant decrease in AOLS in group I patients is of utmost importance.

The present study has some limitations. First, this singlecentered, retrospective, observational study should be considered with caution owing to the relatively small sample size and associated residual confounding. Second, a number of outcomes were not well-delineated due to unavailability of some laboratory data or incomplete profiles in medical records resulted from the fact that laboratory tests are requested at the discretion of the physicians. Third, only patients with moderate disease were admitted in these two wards of the hospital during this period and enrolled in the study. Hence, RCTs are needed to confirm these findings.

Acknowledgements The authors would like to thank the Baqiyatallah University of Medical Science for the facilities offered during study. Authors appreciate Dr. Pooneh Salari's valuable help in review and direct editing of the article. We would also like to thank Vice Presidency for Science and Technology and Iranian Deputy of Research and Technology, Ministry of Health and Medical Education for their supports.

\section{Compliance with ethical standards}

Conflict of interest The authors declare no conflict of interest.

\section{References}

1. Cui J, Li F, Shi Z-L. Origin and evolution of pathogenic coronaviruses. Nat Rev Microbiol. 2019;17(3):181-92. https:// doi.org/10.1038/s41579-018-0118-9.

2. Zhu N, Zhang D, Wang W, Li X, Yang B, Song J, et al. A novel coronavirus from patients with pneumonia in China, 2019. N Engl J Med. 2020;382:727-33. https://doi.org/10.1056/NEJMoa2001017.

3. World health organization (WHO). Coronavirus disease 2019 (COVID-19): situation report, 70. 2020.

4. Huang C, Wang Y, Li X, Ren L, Zhao J, Hu Y, et al. Clinical features of patients infected with 2019 novel coronavirus in Wuhan. China The Lancet. 2020;395(10223):497-506. https://doi. org/10.1016/S0140-6736(20)30183-5.

5. Shi H, Han X, Jiang N, Cao Y, Alwalid O, Gu J, et al. Radiological findings from 81 patients with COVID-19 pneumonia in Wuhan, China: a descriptive study. Lancet Infect Dis. 2020;20(4):425-34. https://doi.org/10.1016/S1473-3099(20)30086-4.

6. Xu Z, Shi L, Wang Y, Zhang J, Huang L, Zhang C, et al. Pathological findings of COVID-19 associated with acute respiratory distress syndrome. Lancet Respir Med. 2020;8(4):420-2. https://doi.org/10.1016/S2213-2600(20)30076-X.
7. Channappanavar R, Perlman S. Pathogenic human coronavirus infections: causes and consequences of cytokine storm and immunopathology. Seminars in immunopathology. 2017;39:529-39. https://doi.org/10.1007/s00281-017-0629-x.

8. Zhang J, Zhou L, Yang Y, Peng W, Wang W, Chen X. Therapeutic and triage strategies for 2019 novel coronavirus disease in fever clinics. Lancet Respir Med. 2020;8(3):11-2. https://doi.org/10. 1016/S2213-2600(20)30071-0.

9. Yang X, Yu Y, Xu J, Shu H, Liu H, Wu Y, et al. Clinical course and outcomes of critically ill patients with SARS-CoV-2 pneumonia in Wuhan, China: a single-centered, retrospective, observational study. Lancet Respir Med. 2020;8(5:475-81. https://doi.org/10. 1016/S2213-2600(20)30079-5.

10. Wu C, Chen X, Cai Y, Zhou X, Xu S, Huang H, et al. Risk factors associated with acute respiratory distress syndrome and death in patients with coronavirus disease 2019 pneumonia in Wuhan, China. JAMA Intern Med. 2020. https://doi.org/10.1001/ jamainternmed.2020.0994.

11. Shi Y, Wang Y, Shao C, Huang J, Gan J, Huang X, et al. COVID19 infection: the perspectives on immune responses. Cell Death \& Differentiation. 2020;27:1451-4. https://doi.org/10.1038/s41418020-0530-3.

12. Mehta P, McAuley DF, Brown M, Sanchez E, Tattersall RS, Manson JJ. COVID-19: consider cytokine storm syndromes and immunosuppression. Lancet. 2020;395(10229):1033-4. https:// doi.org/10.1016/S0140-6736(20)30628-0.

13. Chen N, Zhou M, Dong X, Qu J, Gong F, Han Y, et al. Epidemiological and clinical characteristics of 99 cases of 2019 novel coronavirus pneumonia in Wuhan, China: a descriptive study. Lancet. 2020;395(10223):507-13. https://doi.org/10.1016/ S0140-6736(20)30211-7.

14. Ma J, Xia P, Zhou Y, Liu Z, Zhou X, Wang J, et al. Potential effect of blood purification therapy in reducing cytokine storm as a late complication of severe COVID-19. Clinical Immunology (Orlando, Fla). 2020; 214: 1521-6616. https://doi.org/10.1016/j.clim.2020. $108408 \mathrm{R}$

15. Wu F, Zhao S, Yu B, Chen Y-M, Wang W, Song Z-G, et al. A new coronavirus associated with human respiratory disease in China. Nature. 2020;579(7798):265-9. https://doi.org/10.1038/s41586020-2008-3.

16. R-c C, X-p T, Tan S-y, Liang B-1, Wan Z-y, J-q F, et al. Treatment of severe acute respiratory syndrome with glucosteroids: the Guangzhou experience. Chest. 2006;129(6):1441-52. https://doi. org/10.1378/chest.129.6.1441.

17. Russell CD, Millar JE, Baillie JK. Clinical evidence does not support corticosteroid treatment for $2019-\mathrm{nCoV}$ lung injury. Lancet. 2020;395(10223):473-5. https://doi.org/10.1016/S0140-6736(20) 30317-2.

18. Ruan Q, Yang K, Wang W, Jiang L, Song J. Clinical predictors of mortality due to COVID-19 based on an analysis of data of 150 patients from Wuhan, China. Intensive Care Med. 2020;46:846-8. https://doi.org/10.1007/s00134-020-06028-z.

19. Choudhary R, Sharma AK. Potential use of hydroxychloroquine, ivermectin and azithromycin drugs in fighting COVID-19: trends, scope and relevance. New Microbes and New Infections. 2020;22: 100684.

20. Zheng C, Wang J, Guo H, Lu Z, Ma Y, Zhu Y, et al. Risk-adapted treatment strategy for COVID-19 patients. Int J Infect Dis. 2020;94: 74-7. https://doi.org/10.1016/j.ijid.2020.03.047.

21. Shojaei A, Salari P. COVID-19 and off label use of drugs: an ethical viewpoint. DARU Journal of Pharmaceutical Sciences. 2020;8:1-5. https://doi.org/10.1007/s40199-020-00351-y.

22. Iranian Ministry of health and Medical Education, Diagnostic and therapeutic flowchart of COVID 19 disease at the outpatient and inpatient service levels. national guidelines for novel corona virus. 1 th version; February 28, 2020. 
23. World Health Organization (WHO. a. Clinical management of severe acute respiratory infection when novel coronavirus (nCoV) infection is suspected: interim guidance. 2020.

24. Sun X, Wang T, Cai D, Hu Z, Liao H, Zhi L, et al. Cytokine storm intervention in the early stages of COVID-19 pneumonia. Cytokine Growth Factor Rev. 2020. https://doi.org/10.1016/j.cytogfr.2020. 04.002 .

25. Lescure F-X, Bouadma L, Nguyen D, Parisey M, Wicky P-H, Behillil S, et al. Clinical and virological data of the first cases of COVID-19 in Europe: a case series. Lancet Infect Dis. 2020. https://doi.org/10.1016/S1473-3099(20)30200-0.

26. Zou L, Ruan F, Huang M, Liang L, Huang H, Hong Z, et al. SARSCoV-2 viral load in upper respiratory specimens of infected patients. N Engl J Med. 2020 Mar 19;382(12):1177-9. https://doi. org/10.1056/NEJMc2001737.

27. Cheng PK, Wong DA, Tong LK, et al. Viral shedding patterns of coronavirus in patients with probable severe acute respiratory syndrome. Infect Dis Clin Pract. 2004;12(6):387. https://doi.org/10. 1097/01.idc.0000144912.27311.19.

28. Zhou Z-G, Xie S-M, Zhang J, Zheng F, Jiang D-X, Li K-Y, et al. Short-Term Moderate-Dose Corticosteroid Plus Immunoglobulin Effectively Reverses COVID-19 Patients Who Have Failed LowDose Therapy. Preprints (www.preprints.org). 2020. https://doi. org/10.20944/preprints202003.0065.v1.

29. Shang L, Zhao J, Hu Y, Du R, Cao B. On the use of corticosteroids for 2019-nCoV pneumonia. Lancet (London, England). 2020;395((10225)):683-4. https://doi.org/10.1016/S01406736(20)30361-5.

30. Li H, Chen C, Hu F, Wang J, Zhao Q, Gale RP, et al. Impact of corticosteroid therapy on outcomes of persons with SARS-CoV-2, SARS-CoV, or MERS-CoV infection: a systematic review and meta-analysis. Leukemia. 2020;5:1-9. https://doi.org/10.1038/ s41375-020-0848-3.

31. Yang Z, Liu J, Zhou Y, Zhao X, Zhao Q, Liu J. The effect of corticosteroid treatment on patients with coronavirus infection: a systematic review and meta-analysis. Journal of infection Infect. 2020;30163-4453(20):30191-2. https://doi.org/10.1016/j.jinf. 2020.03.062.

32. Golchin A, Seyedjafari E, Ardeshirylajimi A. Mesenchymal stem cell therapy for COVID-19: present or future. Stem Cell Rev Rep. 2020;13:1-7. https://doi.org/10.1007/s12015-020-09973-w.

33. Cao B, Wang Y, Wen D, Liu W, Wang J, Fan G, et al. A trial of lopinavir-ritonavir in adults hospitalized with severe Covid-19. N Engl J Med. 2020;382:1787-99. https://doi.org/10.1056/ NEJMoa2001282.

34. Stower H. Lopinavir-ritonavir in severe COVID-19. Nat Med. 2020;26(4):465. https://doi.org/10.1038/s41591-020-0849-9.

35. Baden LR, Rubin EJ. Covid-19 - the search for effective therapy. $\mathrm{N}$ Eng1 J Med. 2020;382:1851-2. https://doi.org/10.1056/ NEJMe2005477.
36. Lejal N, Tarus B, Bouguyon E, Chenavas S, Bertho N, Delmas B, et al. Structure-based discovery of the novel antiviral properties of naproxen against the nucleoprotein of influenza a virus. Antimicrob Agents Chemother. 2013;57(5):2231-42. https://doi.org/10.1128/ AAC.02335-12.

37. Micallef J, Soeiro T, Annie-Pierre JB. Non-steroidal anti-inflammatory drugs, pharmacology, and COVID-19 infection. Therapies. 2020;8. https://doi.org/10.1016/j.therap.2020.05.003.

38. Gabriels J, Saleh M, Chang D, Epstein LM. Inpatient use of Mobile continuous telemetry for COVID-19 patients treated with Hydroxychloroquine and azithromycin. HeartRhythm Case Reports. 2020;6(5):241-3. https://doi.org/10.1016/j.hrcr.2020.03. 017.

39. Assiri A, Al-Tawfiq JA, Al-Rabeeah AA, Al-Rabiah FA, Al-Hajjar S, Al-Barrak A, et al. Epidemiological, demographic, and clinical characteristics of 47 cases of Middle East respiratory syndrome coronavirus disease from Saudi Arabia: a descriptive study. Lancet Infect Dis. 2013;13(9):752-61. https://doi.org/10.1016/ S1473-3099(13)70204-4.

40. Lee N, Hui D, Wu A, Chan P, Cameron P, Joynt GM, et al. A major outbreak of severe acute respiratory syndrome in Hong Kong. $\mathrm{N}$ Engl J Med. 2003;348(20):1986-94. https://doi.org/10.1056/ NEJMoa030685.

41. Litao MKS, Kamat D. Erythrocyte sedimentation rate and Creactive protein: how best to use them in clinical practice. Pediatr Ann. 2014;43(10):417-20. https://doi.org/10.3928/0090448120140924-10

42. Wang Z, Chen X, Lu Y, Chen F, Zhang W. Clinical characteristics and therapeutic procedure for four cases with 2019 novel coronavirus pneumonia receiving combined Chinese and Western medicine treatment. Bioscience trends. 2020;14(1):64-8. https://doi.org/ 10.5582/bst.2020.01030.

43. Cui W, Fan Y, Wu W, Zhang F. Wang J-y, Ni A-p. expression of lymphocytes and lymphocyte subsets in patients with severe acute respiratory syndrome. Clin Infect Dis. 2003;37(6):857-9. https:// doi.org/10.1086/378587.

44. Li T, Qiu Z, Zhang L, Han Y, He W, Liu Z, et al. Significant changes of peripheral $\mathrm{T}$ lymphocyte subsets in patients with severe acute respiratory syndrome. J Infect Dis. 2004;189(4):648-51. https://doi.org/10.1086/381535.

45. Kim KD, Zhao J, Auh S, Yang X, Du P, Tang H, et al. Adaptive immune cells temper initial innate responses. Nat Med. 2007;13(10):1248-52.

Publisher's note Springer Nature remains neutral with regard to jurisdictional claims in published maps and institutional affiliations. 